\title{
eJRIEPS
}

Ejournal de la recherche sur l'intervention en éducation physique et sport

12 | 2007

Varia

\section{Formation au Managérat Réciproque en tennis de table chez des élèves de 14-15 ans : tentative de modélisation et mesure des effets}

\section{Pierre Ensergueix et Lucile Lafont}

\section{(2) OpenEdition}

1 Journals

\section{Édition électronique}

URL : https://journals.openedition.org/ejrieps/6173

DOI : 10.4000 /ejrieps. 6173

ISSN : 2105-0821

Éditeur

ELLIADD

\section{Référence électronique}

Pierre Ensergueix et Lucile Lafont, « Formation au Managérat Réciproque en tennis de table chez des élèves de 14-15 ans : tentative de modélisation et mesure des effets ", eJRIEPS [En ligne], 12 | 2007, mis en ligne le 01 juillet 2007, consulté le 27 juin 2021. URL : http://journals.openedition.org/ejrieps/ 6173 ; DOI : https://doi.org/10.4000/ejrieps.6173

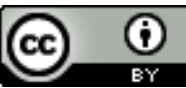

La revue eJRIEPS est mise à disposition selon les termes de la Creative Commons Attribution 4.0 International License. 
Formation au Managérat Réciproque en tennis de table chez des élèves de 14-15 ans : tentative de modélisation et mesure des effets.

\section{Pierre Ensergueix \& Lucile Lafont}

Composante VSTII Laboratoire «Cultures, Education, Sociétés », LACES, EA 4041, Université Victor Segalen Bordeaux2, Faculté des Sciences du Sport, 12 avenue C. Jullian, 33607 Pessac cedex

\section{Résumé}

Le but de cette étude est de contribuer à l'identification des conditions d'efficacité des stratégies d'apprentissage coopératif. Pour ce faire, elle se propose de modéliser et d'examiner les effets d'une Formation au Managérat Réciproque entre Pairs (FMRP) sur l'acquisition de compétences motrices et décisionnelles en tennis de table. Les participants, 36 garçons et 38 filles de 14-15 ans, ont été répartis, sous forme de dyades symétriques, en (3 $\times$ 2) groupes en fonction de la condition d'entraînement : Managérat Formé (MF) vs Managérat Spontané (MS) vs pratique seule (Groupe Contrôle, GC) et en fonction du genre : Garçons vs Filles.

Les résultats sont en partie conformes aux attentes : on observe un gain d'efficacité et de lucidité dans la réalisation de la tâche-cible uniquement chez les participants ayant bénéficié de l'aide éclairée d'un pair (MF). Plus qu'essentielle, la FMRP s'avère une condition d'efficacité coopérative indispensable. Par ailleurs, l'existence d'une sensibilité plus importante des filles à la structuration de leur environnement social est confirmée dans le cadre d'acquisitions motrices faiblement connotées sexuellement.

Note : Afin d'inscrire cette recherche dans un contexte à la fois éducatif et sportif, les termes «tutorat » et « managérat » sont employés par les auteurs comme des synonymes, ne reflétant en aucune manière leurs convictions idéologiques ou politiques.

\section{Introduction}

Cette étude s'inscrit dans un programme de recherche s'intéressant aux apports de la psychologie sociale du développement et des acquisitions au domaine de l'intervention pédagogique en Education Physique et Sportive (EPS).

Support privilégié de l'EPS, les Activités Physiques et Sportives (APS) offrent aux élèves de nombreuses occasions d'interagir : contribuer au gain d'une rencontre en sport collectif, 
participer à un relais en athlétisme, réaliser une parade en gymnastique, assurer un partenaire en escalade... (Lafont \& Winnykamen, 1999). Dès lors, plusieurs études, conduites notamment au sein de l'équipe «Vie Sportive, Trajectoires, Innovation, Intervention », LACES EA 4140, s'interrogent sur l'efficacité respective de différents modes d'intervention et d'interactions sociales originellement développés dans le cadre d'acquisitions de tâches académiques. Regroupées sous le terme générique d'Apprentissage Coopératif (AC) en Europe et de Cooperative Learning (CL) aux EtatsUnis (Duran \& Monereo, 2005), ces procédures sont considérées aujourd'hui comme d'utiles alternatives à l'enseignement traditionnel. En proposant à l'apprenant d'évoluer en dyade ou en groupe restreint, elles lui procurent un rôle essentiel d'acteur, favorisant les déséquilibres et restructurations nécessaires à l'apprentissage. Au demeurant, ces stratégies semblent constituer une réponse possible aux difficultés induites par l'hétérogénéité croissante des élèves et permettre à l'enseignant d'être plus disponible à l'égard de certains élèves et d'assumer d'autres fonctions clés (Lafont, Cicero, Vedel \& Viala, 2005). L'obtention souvent conjointe de bénéfices cognitifs, motivationnels, sociaux et moteurs est mentionnée dans la majorité des études relatives à l'AC dans le champ des habiletés motrices (Ward \& Lee, 2005).

De nombreux enseignants d'EPS considèrent implicitement que le seul fait de permettre à des élèves de travailler en équipe suffit à ce qu'ils apprennent en coopération, alors qu'en réalité de nombreux jeux favorisent davantage la compétition interindividuelle. Le simple fait de mettre des élèves en groupe et de leur demander de travailler ensemble n'aboutit que très rarement à une véritable coopération. Au contraire, l'absence de structuration de l'AC conduit souvent à des situations compétitives entre les groupes, à des efforts individuels avec simple discussion entre les membres et parfois même à une dégradation de la performance des élèves (Johnson, Johnson \& Smith, 1998). Plusieurs travaux ont ainsi indiqué la nécessité de faire correspondre l'AC à un aménagement éducatif dans lequel les participants travaillent ensemble dans le but de maîtriser un contenu (Johnson \& Johnson, 1989 ; Kagan, 1990, Slavin, 1995). L'investigation des conditions d'efficacité des stratégies d'AC est devenue depuis peu incontournable. Pour Duran et Monereo (2005), on assiste au passage d'une première génération d'études, centrée sur les effets de l'AC, à une seconde génération d'études, orientée sur l'identification des causes et mécanismes expliquant les bénéfices coopératifs observés.

Parmi ces conditions d'efficacité, la nécessité de faire acquérir aux élèves des habiletés coopératives est fréquemment évoquée. Le partage de connaissances avec un ou plusieurs pairs requiert des compétences souvent absentes du répertoire initial des élèves 
et rarement considérées par les enseignants comme de véritables contenus d'enseignement. Par exemple, en contexte d'interaction de tutelle, des études montrent l'intérêt d'une formation à la fonction de guidage à laquelle les individus sont rarement préparés (Lafont et al., 2005). Ces auteurs font notamment référence aux travaux ayant investigué la problématique de la sensibilité des tuteurs enfants comparativement à celle des adultes. Des différences significatives ont été observées en faveur des tuteurs adultes et peuvent s'expliquer par les trois éléments suivants : la gestion matérielle de la tâche, la gestion des informations données aux partenaires et la gestion sociale des interactions (Ellis \& Rogoff, 1982). Comme le rappelle Winnykamen (1996), il ne suffit pas d'être expert dans une tâche pour être un bon tuteur : le tuteur est toujours un expert, l'inverse n'est pas systématique.

Outre l'étude des conditions favorables à l'émergence de bénéfices variés chez les apprenants, la recherche inhérente aux procédures d'AC semble s'intéresser de plus en plus à la tutelle réciproque entre pairs (Reciprocal Peer Tutoring, RPT). Ainsi, le recours à un tutorat fixe permettant à un élève novice (tutoré) de recevoir une aide de la part d'un élève expert souvent plus âgé (tuteur) laisse progressivement sa place à un tutorat réciproque présentant à l'inverse une forte symétrie dans les dyades (Duran et Monereo, 2005). Développé au départ par Fantuzzo et ses collègues (1989, 1992, 1997), la procédure RPT est née en réponse au besoin grandissant de découvrir des stratégies efficaces, susceptibles d'aider les enfants issus de milieux défavorisés à se sentir compétents sur le plan scolaire et à réaliser des progrès. L'avantage du RPT par rapport aux autres stratégies d'AC est triple. D'une part, il étend les bénéfices d'un tutorat fixe aux deux membres de la dyade tout en réduisant ses inconvénients (autoritarisme, dépendance au tuteur...). D'autre part, il incorpore certains avantages du CL tout en minimisant ses désavantages (comportement d'évitement du rôle de médiateur, risque de régression pour un élève expert...). De plus, il est particulièrement approprié aux objectifs du contexte scolaire : l'apprentissage de tous les élèves. Jouant alternativement le rôle de tuteur et de tutoré, le RPT permet aux deux apprenants de récolter les bénéfices liés à la préparation à l'enseignement, l'évaluation et le soutien d'un pair. La stratégie RPT semble par conséquent plus adaptée aux conditions écologiques de la classe ainsi qu'aux objectifs de méthodes, d'attitudes et de savoirs faire sociaux très présents dans les programmes actuels du second degré. Toutefois, si l'essor du RPT semble effectif dans le champ des disciplines académiques, son potentiel pédagogique demeure peu exploité dans celui des habiletés motrices, notamment en EPS. Pour preuve, la méta-analyse de Ward et Lee (2005), faisant état de la recherche inhérente à l'AC en EPS, ne recense que 
neuf études relatives au RPT. De plus, celles-ci se contentent souvent d'évaluer les effets de la procédure réciproque en omettant d'investiguer les conditions de son efficacité.

Par ailleurs, l'intérêt grandissant porté au RPT place la question de l'effet du genre sur les bénéfices attendus au cœur des problématiques actuelles relatives à l'AC. En effet, à la symétrie d'âge et de compétence affichée dans les dyades RPT vient s'ajouter également celle du genre. Dès lors, eu égard à la tendance des études de deuxième génération mentionnées en amont, des comparaisons entre dyades filles et dyades garçons semblent pertinentes. Concernant le domaine de l'intervention en EPS, la problématique des différences liées au genre a été appréhendée au sein d'interactions de tutelle non réciproques (Arripe-Longueville (d'), Gernigon, Huet, Cadopi \& Winnykamen, 2002 ; Legrain, Arripe-Longueville (d') \& Gernignon, 2003) ou en classe entière (Classwide Peer Tutoring, CWPT, Johnson \& Ward, 2001). Les résultats de ces études laissent penser que les plus grands bénéficiaires diffèrent selon les variables considérées. D'une part, en accord avec les travaux issus du champ des disciplines académiques (Charlesworth \& Dzur, 1987), les filles affichent un ensemble de conduites plus favorables aux situations d'AC que les garçons. Par exemple, dans une tâche de virage brasse en natation, les travaux de Arripe-Longueville (d') et al. (2002) montrent un nombre de verbalisations plus élevé au sein des dyades féminines tandis qu'un fort engagement physique dans la tâche caractérise davantage les dyades masculines. D'autre part, les garçons présentent des performances motrices et un Sentiment d'Efficacité Personelle (SEP, Bandura, 1997) plus importants que les filles. Toutefois, ces conclusions sont nuancées par celles d'études plus récentes. Ainsi, aucune différence significative relativement à la nature des conduites adoptées dans les dyades (Legrain et al., 2003) et aux performances motrices obtenues par les élèves (Johnson \& Ward, 2001) n'est constatée. Cette absence d'effet du genre pourrait, selon ces auteurs, être corrélée avec le caractère très structuré des procédures interactives employées.

La présente étude souhaite apporter une contribution aux problématiques actuelles relatives à l'AC dans le domaine de l'intervention en EPS. Ainsi, elle tente d'identifier les conditions favorables à l'émergence de bénéfices variés dans le cadre d'une procédure $R P T$. Au plan opérationnel, trois objectifs sont envisagés. Premièrement, il s'agit de proposer une modélisation, pour des élèves débutants impliqués dans une pratique physique (tennis de table), d'un programme de Formation au Managérat Réciproque entre Pairs (FMRP). Deuxièmement, l'étude consiste à comparer les conséquences sur les performances et progrès moteurs de ce programme de formation à celles générées par 
l'exercice d'un managérat spontané et/ou par l'absence de managérat. Troisièmement, d'éventuelles différences liées au genre seront envisagées à titre exploratoire.

L'hypothèse principale de cette étude postule que le suivi par les élèves du programme FMRP est une condition d'efficacité essentielle dans l'attente raisonnée de bénéfices moteurs en tennis de table. Par ailleurs, une hypothèse exploratoire concernant l'existence d'un effet du genre sur les progrès moteurs réalisés en situation d'interaction dyadique réciproque est formulée. Ainsi, l'absence de réciprocité des stratégies d'AC et la forte connotation de genre des APS retenues dans les études antérieures (Arripe-Longueville (d') et al., 2002 ; Legrain et al., 2003) invitent à considérer les conclusions de ces dernières avec précaution.

\section{Méthode}

\subsection{Participants}

Soixante quatorze participants âgés de 15,3 ans $(S D=1,3), 38$ filles et 36 garçons, sélectionnés à partir de la population de trois classes de $3^{\text {ème }}$ d'un collège français de milieu urbain (90 élèves), ont pris part de manière volontaire à l'expérimentation. Une autorisation écrite des parents à participer à l'expérimentation pour chacun des élèves a été obtenue par le chef d'établissement. Les élèves sont de niveau débutant dans l'activité tennis de table avec un vécu antérieur de 10 heures.

\subsection{Plan expérimental}

Un plan factoriel $3 \times 2 \times 3$ est utilisé. Le premier facteur concerne la condition d'entraînement: Managérat Formé + pratique physique (MF) vs Managérat Spontané + pratique physique pratique (MS) vs pratique physique seule (Groupe Contrôle, GC) ; le deuxième facteur renvoie au genre: garçons vs filles; le troisième facteur concerne le temps : pré-test (PT), post-test immédiat (PO) et post-test différé (PD).

Après avoir contrôlé le niveau de compétence motrice initiale spécifique à la tâche expérimentale lors du PT (cf. Procédure), les 74 participants ont été répartis aléatoirement, sous forme de dyades non mixtes, en six groupes indépendants.

\subsection{Tâche-cible et dispositif matériel}

La tâche-cible est appelée «La double attention » en référence à deux situations d'apprentissage distinctes (Delisle, 1999 ; Sève, 2003). Conçue en accord avec un spécialiste de l'activité tennis de table, elle est de type technico-tactique.

Concrètement, un expert en tennis de table envoie, à intervalle de temps régulier, 20 balles directement et aléatoirement sur la demi-table du participant. La zone de rebond et la hauteur de la balle varient par conséquent à chaque fois. Le participant doit réagir et 
renvoyer la balle après s'être déplacé. Son objectif est d'obtenir le meilleur score possible. Pour cela, il doit tenir compte d'un système de comptage de points particulier (cf. tableau 1). Selon la nature de son renvoi, il peut obtenir entre 0 et 6 points. Une bonification est attribuée si le type de frappe est pertinent eu égard au type de trajectoire de balle reçue. La situation est neutre dans la mesure où l'expert n'est pas directement concerné par la réussite ou l'échec du participant. Un aménagement de la table (cf. fig. 1) est retenu afin de faciliter la compréhension des buts de la tâche et notamment de la grille de score. Le joueur dispose de deux essais et d'un temps-mort d'une minute suite à son premier essai. Enfin, L'enregistrement filmé est assuré par la présence d'une caméra numérique placé à une distance de 3 mètres de l'action sur un trépied. La prise de vue est réalisée de côté et sur un plan surélevé afin de faciliter la prise en compte à la fois des trajectoires de balle produites (i.e., par l'expert et par le joueur) et des déplacements du joueur.

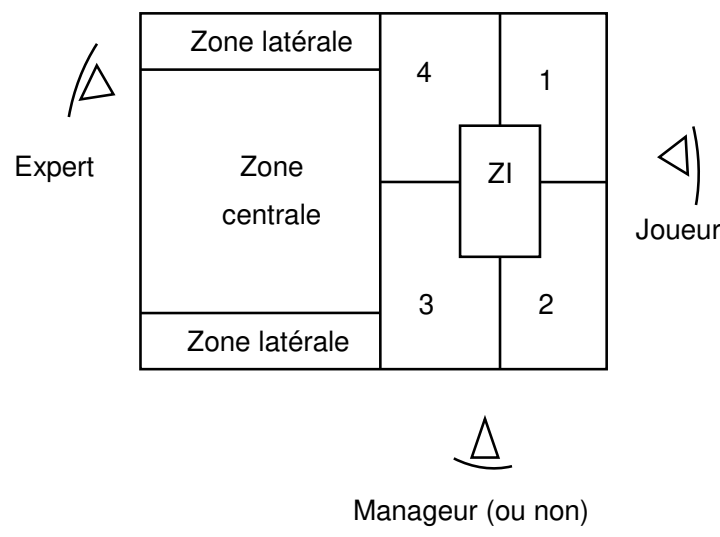

Tableau 1. Grille de score

\begin{tabular}{|c|c|c|c|c|c|}
\hline Point(s) & 0 & 1 & 2 & 3 & Bonus + 2 \\
\hline $\begin{array}{c}\text { Renvoi } \\
\text { simple }\end{array}$ & Non renvoi & $\begin{array}{c}\text { - Dehors } \\
- \text { Filet }\end{array}$ & $\begin{array}{c}\text { Zone } \\
\text { centrale }\end{array}$ & $\begin{array}{c}\text { Zones } \\
\text { latérales }\end{array}$ & $\begin{array}{c}\text { Si balle } \\
\text { tendue }\end{array}$ \\
\hline Point(s) & 0 & 2 & 4 & 6 & Bonus + 2 \\
\hline Smash & $\begin{array}{c}\text { Non renvoi } \\
\text { (geste) }\end{array}$ & $\begin{array}{c}\text { - Dehors } \\
\text { - Filet }\end{array}$ & $\begin{array}{c}\text { Zone } \\
\text { centrale }\end{array}$ & $\begin{array}{c}\text { Zones } \\
\text { latérales }\end{array}$ & $\begin{array}{c}\text { Si balle } \\
\text { haute }\end{array}$ \\
\hline
\end{tabular}

Figure 1. Tâche-cible

\subsection{Procédure}

L'expérience a pris place au sein d'un cycle de tennis de table de sept séances de deux heures et se déroule selon 5 phases successives: (a) montante-descendante initiale (MD1) et pré-test (PT) lors de la première séance, (b) entraînement selon trois conditions (MF, MS et GC) pendant les 4 séances suivantes, (c) post-test immédiat ( $P O)$ à la sixième séance, et (d) post-test différé (PD) et montante-descendante finale (MD2) au cours de la septième séance. L'étude a été conduite pendant les leçons d'EPS, dans un espace spécifiquement dévolu à l'expérimentation, de façon à ce que les participants ne puissent pas s'observer d'un groupe à l'autre. Ainsi, chacun des groupes de 4 élèves (2 dyades) venaient successivement réaliser le protocole. Par ailleurs une consigne de confidentialité de la formation a été donnée dans le but d'éviter toute influence entre les différents groupes. 
La $1{ }^{\text {ère }}$ séance a permis d'évaluer le niveau initial des élèves en situation réelle de match et dans la tâche-cible. Tout d'abord, afin de mesurer d'éventuels effets de la condition d'entraînement et/ou du genre sur les performances des participants en situation concrète de jeu, ces derniers ont réalisé une série de 7 matchs consécutifs de 4 minutes (MD1, cf. Mesures). Puis, dans le but de constituer des dyades relativement symétriques et d'écarter de l'étude les participants manifestant un niveau de pratique trop élevé/faible, chacun d'entre eux a effectué le PT en réalisant la tâche-cible décrite précédemment. L'exécution motrice était alors filmée afin de pouvoir, a posteriori, apprécier finement le score de compétence motrice par un double jugement d'experts. Seuls les participants présentant un score de compétence motrice compris entre 70 et 170 ont été retenus (cf. mesures). Sur la base de ces scores, un classement a été réalisé dans chacune des classes. Le principe retenu était d'associer le participant du rang le plus élevé avec celui, du même genre, classé juste en dessous. Par ailleurs, l'avis de l'enseignant a été également sollicité, évitant ainsi les appareillages entre participants présentant un niveau trop élevé de rejet ou d'affinité. Enfin, les dyades ont été affectées aléatoirement à l'une des trois conditions expérimentales (MF, MS et GC).

Les 4 séances suivantes proposaient à tous les participants de suivre un programme de pratique physique de quatre séquences de 3 minutes afin de confronter les élèves à la tâche-cible un nombre suffisant de fois pour espérer l'émergence de progrès en fin de cycle.

Suivi seul par les participants de la condition GC, ce programme a été complété par l'exercice d'une fonction managérale pour les participants des conditions interactives MF et MS. Celle-ci s'intégrait dans la séquence de pratique physique en offrant la possibilité aux participants d'endosser à la fois le rôle de joueur et celui de manageur. A leur arrivée à la table, chacun des membres de la dyade se voyait attribuer le rôle de joueur ou de manageur. Le joueur réalisait la tâche-cible selon les mêmes modalités que celles présentées au PT. Au cours de la tâche, le manageur devait observer son partenaire en silence. Pendant le temps-mort, les participants étaient en interaction et libres d'interagir à leur guise. A l'issue des deux essais du premier joueur, les rôles étaient inversés.

Les participants de la condition MF ont exercé leur fonction managériale dans des conditions identiques à celles de la condition MS. En revanche, ils ont reçu une formation à cette fonction. Le dispositif de cette Formation au Managérat Réciproque d'un Pair (FMRP) comportait deux séquences de $60 \mathrm{~min}$, réalisées en dehors des cours d'EPS, entre la première et la deuxième séance du cycle. Ses composantes méthodologiques s'inspiraient de quatre études menées dans le champ des disciplines académiques et 
motrices. Ainsi, conformément aux travaux de Lorence (2002), elle visait à créer un « contexte communicationnel » entre l'expérimentateur et les apprenants afin de permettre une co-construction du sens et des buts de la formation. De plus, en référence à l'étude de Wiegmann, Dansereau \& Patterson (1992), la tâche était divisée en trois sous-tâches dans le but de faciliter le travail d'observation des apprenants. Egalement, en continuité avec les expérimentations conduites par Lafont et al. (2005), des règles de fonctionnement d'un bon manageur ont été expliquées aux participants. Enfin, en prenant exemple sur la Technique du Groupe Nominal (TGN) développée par Brunelle et al. (1988), la participation active de l'ensemble des membres de la condition MF était sollicitée. L'objectif de cette formation était de faire émerger et intégrer chez les participants, d'une part, les critères de réalisation et de réussite d'une tâche spécifique de tennis de table, et, d'autre part, les règles de fonctionnement d'un bon manageur.

La $6^{\text {ème }}$ séance correspondait à la réalisation du $\mathrm{PO}$, de manière identique au PT pour les participants de la condition GC et en dyade pour ceux des conditions interactives.

La $7^{\text {ème }}$ séance, réalisée deux semaines après le $P O$, a mesuré la stabilité des résultats obtenus au PO grâce à un PD et a évalué le niveau final des élèves en situation concrète de match à l'aide d'une deuxième montante-descendante (MD2), identique à MD1.

\subsection{Mesures}

Les performances et progrès moteurs font l'objet d'une double évaluation : en situation concrète de jeu à l'aide d'un score de montante-descendante (MD) et relativement à la tâche-cible grâce à un score de compétence motrice (CM).

Concernant MD, il correspond à la différence entre le numéro de table atteint par le joueur à l'issue de la montante-descendante initiale (MD1) et celui atteint à la montantedescendante finale (MD2). Une montante-descendante se caractérise par : (a) une répartition aléatoire des joueurs sur 8 tables cotées préalablement selon 4 niveaux, (b) la réalisation par chacun des joueurs de 7 matchs de 4 minutes au cours desquels il « monte » d'une table en cas de victoire et « descend » d'une table après une défaite. Par conséquent, MD s'échelonne de -3 à +3 .

Conformément aux travaux de Darnis, Lafont \& Menaut (2005a, 2005b), le score de compétence motrice $(\mathrm{CM})$, correspond à deux indicateurs de compétence motrice en situation décisionnelle : un score d'efficacité de l'action (EA) et un score de pertinence de choix (PC). Compte tenu de certaines spécificités de la tâche-cible, le calcul de ces scores est obtenu à partir du support vidéo et sa fiabilité est assurée grâce à un double codage d'experts. Par rapport à EA, selon le type de frappe réalisé et la zone d'impact de la balle, les participants obtiennent à chaque renvoi un score compris entre 0 et 6 . Parmi les 20 
balles envoyées par l'expert au cours d'un essai, 7 ont une trajectoire haute sur laquelle il est possible de smasher et 13 ont une trajectoire tendue sur laquelle il est difficile voire impossible de réussir un smash. Afin de limiter les abus, un smash réussi sur une balle tendue est assimilé à un renvoi simple. Ainsi, en additionnant les scores des 40 renvois effectués par le joueur au cours de ses deux essais, EA s'échelonne de 0 à 162. Relativement à PC, en fonction de l'adéquation type de frappe effectué/trajectoire de la balle reçue, un bonus de 2 points par renvoi est attribué. Par conséquent, PC varie sur une échelle allant de 0 à 80.

\subsection{Analyse des données}

Afin de tester l'hypothèse selon laquelle les participants de la condition MF manifestent des performances et des progrès dans la réalisation de la tâche-cible (i.e., gain d'efficacité et de lucidité) plus élevés comparativement à ceux des conditions MS et GC et observer d'éventuelles différences liées au genre, une analyse de variance (ANOVA) 3 × 2 × 3 (Condition d'entraînement $x$ Genre $x$ Temps) à mesures répétées sur le dernier facteur (RMANOVA) est conduite sur le score d'efficacité de l'action et le score de pertinence de choix. S'agissant du score de montante-descendante, une ANOVA 3 × 2 (Condition d'entraînement x Genre) est réalisée.

\section{Résultats}

3. 1. Score de montante-descendante

Tableau 2 - Moyennes et (écarts-types) par conditions et genres à la mesure de la montantedescendante

\begin{tabular}{lll}
\hline Condition & Genre & \\
\hline \multirow{2}{*}{ Managérat Formé } & Filles & $0,4(0,51)$ \\
& Garçons & $0,1(0,67)$ \\
Managérat & Filles & $-0,3(0,65)$ \\
Spontané & Garçons & $0,0(0,74)$ \\
Groupe Contrôle & Filles & $-0,2(0,80)$ \\
& Garçons & $-0,2(1,03)$ \\
\hline
\end{tabular}

L'ANOVA effectuée sur cette variable révèle une tendance à la significativité de l'effet de la condition d'entraînement, $F(2,68)=2,64, p=0,079$. Le test post-hoc de Bonferroni montre que les participants de la condition MF obtiennent un score plus élevé que ceux de la condition MS et GC. En revanche, aucune différence n'est constatée entre les participants de la condition MS et les participants de la condition GC. Par ailleurs, les résultats de l'ANOVA montrent l'absence d'effet principal lié au genre et à l'interaction entre la 
condition d'entraînement et le genre. Cependant, l'analyse des statistiques descriptives (cf. tableau 3) montre que les filles de la condition $\operatorname{MF}(M=0,42, \sigma=0,51)$ affichent un score de montantedescendante plus élevé que celui obtenu par les garçons de la même condition $(M=0,08, \sigma=0,67)$.

\section{2. Score de compétence motrice}

Au pré-test, relativement au score de l'efficacité de l'action et au score de pertinence du choix, on n'observe ni effet principal de la condition d'entraînement ni effet d'interaction entre la condition d'entraînement et le genre. L'homogénéité entre les trois conditions d'entraînement au PT est donc vérifiée. En revanche, les ANOVAs effectuées sur ces variables indiquent un effet principal du genre $(F(1,68)=49,77, p<0,05 ; F(1,68)=19,76, p<0,05)$, les garçons $(M=$ 87,53, $\sigma=8,27 ; M=59,00, \sigma=7,05)$ manifestant des scores plus élevés que les filles $(M$ $=69,95, \sigma=12,47 ; M=51,84, \sigma=7,21)$.

3. 3. Score de l'efficacité de l'action

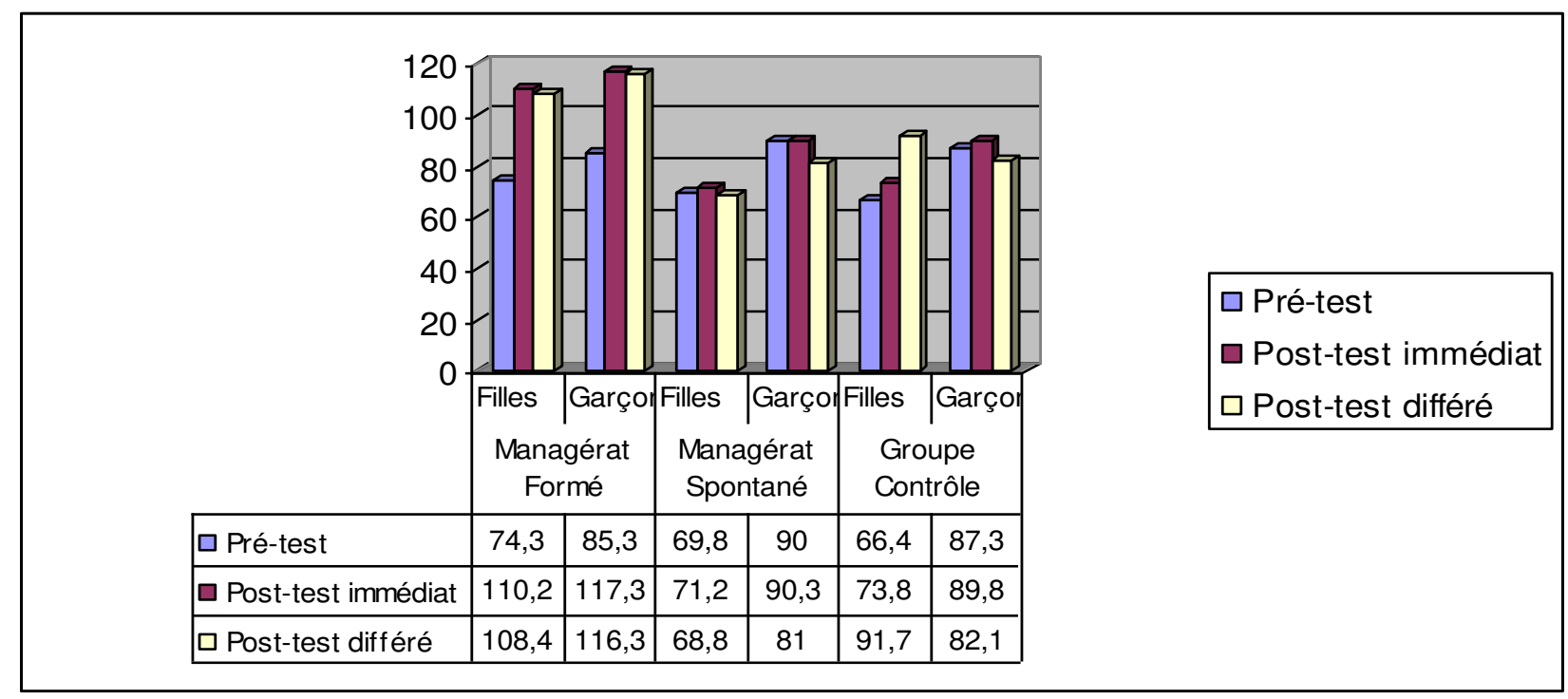

Figure 2 - Moyennes par conditions et genre à la mesure de l'efficacité de l'action -

Au post-test immédiat, l'ANOVA met en évidence un effet principal de la condition d'entraînement, $F(2,68)=86,76, p<0,05$. Le test post-hoc de Bonferroni montre que les participants de la condition MF obtiennent un score significativement plus élevé que ceux de la condition MS et ceux de la condition GC. En revanche, aucune différence significative n'est constatée entre les participants de la condition MS et ceux de la condition GC. Par ailleurs, les résultats de l'ANOVA indiquent un effet principal du genre, $F(1,68)=37,21, p<0,05$, les garçons $(M=99,14, \sigma=15,95)$ manifestant une efficacité de l'action plus élevée que les filles $(M=84,45, \sigma=20,40)$. Enfin, l'ANOVA montre l'absence d'effet d'interaction entre la condition d'entraînement et le genre. 
Au post-test différé, les résultats sont sensiblement identiques. Ainsi, l'ANOVA met en évidence un effet principal de la condition d'entraînement, $F(2,68)=54,57, p<0,05$. Le test post-hoc de Bonferroni montre que les participants de la condition MF ont obtenu un score plus élevé que ceux de la condition MS et ceux de la condition GC. En revanche, aucune différence significative n'est constatée entre les participants de la condition MS et ceux de la condition GC. Par ailleurs, les résultats de l'ANOVA indiquent un effet principal du genre, $F(1,68)=37,41, p<0,05$, les garçons $(M=100,39, \sigma=15,31)$ manifestant une efficacité de l'action significativement plus élevée que les filles $(M=83,16, \sigma=21,47)$. Enfin, l'ANOVA montre une tendance à la significativité de l'effet d'interaction entre la condition d'entraînement et le genre, $F(2,68)=3,07, p=0,053$. Plus précisément, les filles de la condition MF (M=108,42, $\sigma=8,87)$ affichent un score d'efficacité de l'action supérieur à celui obtenu par leurs congénères de la condition $M S(M=68,75, \sigma=14,77)$ et $\mathrm{GC}(M=$ $73,86, \sigma=13,69)$.

Entre le pré-test et le post-test immédiat, RMANOVA montre des progrès significatifs uniquement pour les garçons et les filles de la condition MF. Ainsi, elle indique un effet de l'interaction entre le temps et la condition d'entraînement, $F(2,68)=64,76, p<0,05$, $E S=$ 0,66. Le test post-hoc de Bonferroni montre que les participants de la condition MF progressent plus significativement que ceux de la condition MS $(+16,46)$ et ceux de la condition GC (+ 18,17). En revanche, aucune différence significative n'est constatée entre les participants de la condition MS et ceux de la condition GC. Par ailleurs, aucun effet de l'interaction du temps et $d u$ genre et de la triple interaction temps*condition d'entraînement*genre n'est enregistré.

Entre le pré-test et le post-test différé, RMANOVA présente les mêmes effets que ceux mentionnés précédemment.

3. 4. Score de la pertinence du choix

Au post-test immédiat, l'ANOVA met en évidence un effet principal de la condition d'entraînement, $F(2,68)=51,84, p<0,05$. Le test post-hoc de Bonferroni montre que les participants de la condition MF obtiennent un score significativement plus élevé que ceux de la condition MS et ceux de la condition GC. En revanche, aucune différence significative n'est constatée entre les participants de la condition MS et ceux de la condition GC. Par ailleurs, les résultats de l'ANOVA indiquent une tendance à la significativité de l'effet du genre, $F(1,68)=37,21, p=0,060$, les garçons $(M=65,72, \sigma=$ $10,22)$ manifestant une pertinence du choix plus élevée que les filles $(M=62,42, \sigma=$ 11,35). Enfin, l'ANOVA montre une tendance à la significativité de l'effet d'interaction entre la condition d'entraînement et le genre, $F(2,68)=2,76, p=0,071, E S=0,07$. Plus 
précisément, les filles de la condition MF $(M=75,16, \sigma=4,78)$ affichent un score de la pertinence du choix supérieur à celui obtenu par leurs congénères de la condition $\mathrm{MS}(M=$ $54,33, \sigma=6,87)$ et $\mathrm{GC}(M=58,43, \sigma=8,95)$.

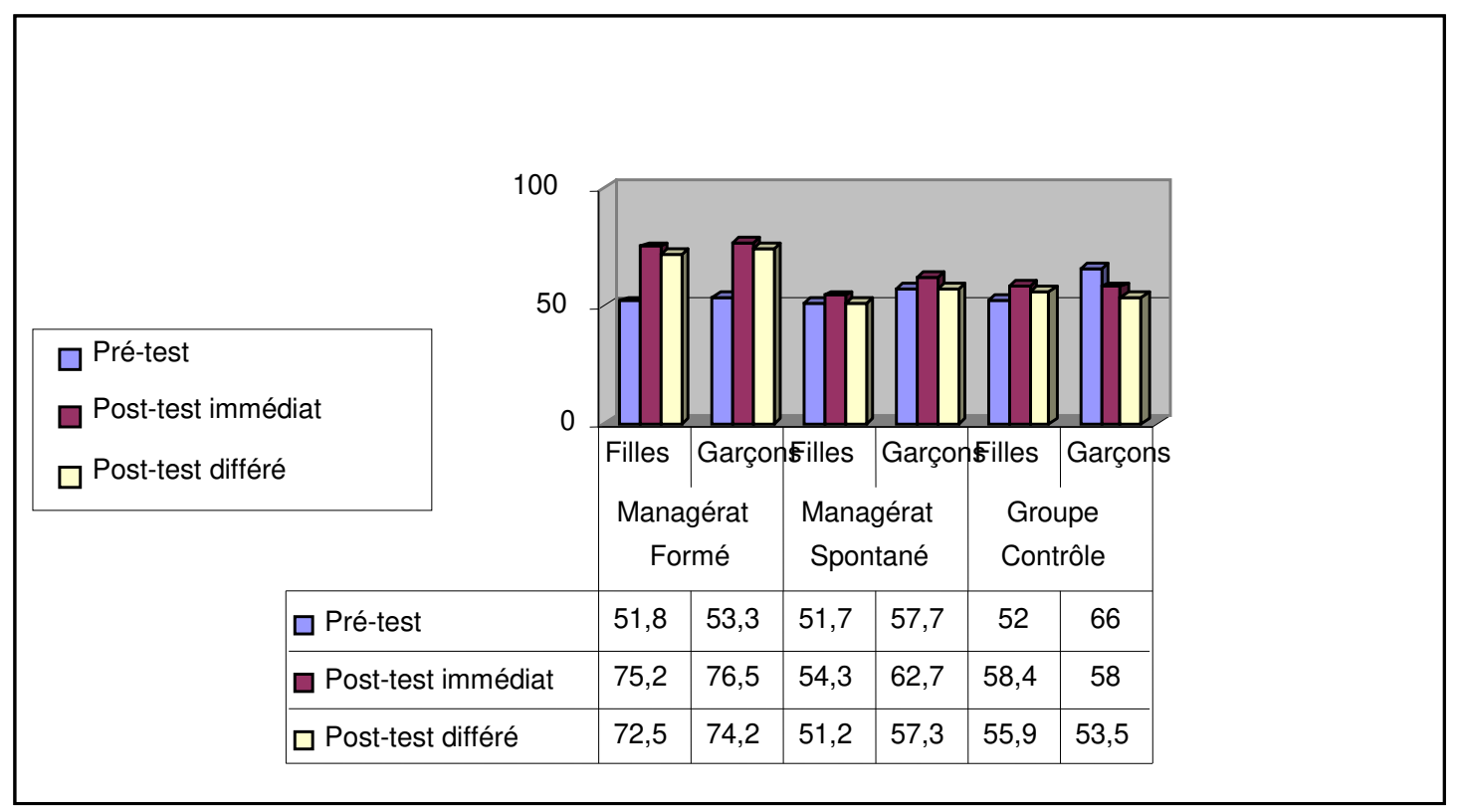

Figure 3 - Moyennes par conditions et genre à la pertinence du choix -

Au post-test différé, l'ANOVA met en évidence un effet principal de la condition d'entraînement, $F(2,68)=48,72$, p<0,05. Le test post-hoc de Bonferroni montre que les participants de la condition MF ont obtenu un score plus élevé que ceux de la condition MS et ceux de la condition GC. En revanche, aucune différence significative n'est constatée entre les participants de la condition MS et ceux de la condition GC. Par ailleurs, les résultats de l'ANOVA n'indiquent aucun effet principal du genre et de l'interaction entre la condition d'entraînement et le genre.

Entre le pré-test et le post-test immédiat, RMANOVA montre des progrès significatifs pour l'ensemble de la population d'étude, $F(1,68)=65,47, p<0,05$. De plus, elle indique un effet de l'interaction entre le temps et la condition d'entraînement, $F(2,68)=46,05$, $p<0,05$. Le test post-hoc de Bonferroni montre que les participants de la condition MF ont progressé plus que ceux de la condition MS et ceux de la condition GC. En revanche, aucune différence significative n'est constatée entre les participants de la condition MS et ceux de la condition GC. Par ailleurs, RMANOVA indique une tendance à la significativité de l'effet d'interaction du temps et du genre, $F(1,68)=37,21, p=0,064$, les filles $(+10,58)$ manifestant un progrès plus important que les garçons $(+6,72)$. Enfin, RMANOVA montre un effet de la triple interaction temps* condition d'entraînement* ${ }^{*}$ enre, $F(2,68)=5,94$, 
$p<0,05$. Plus précisément, les filles de la condition MF ont progressé plus que leurs congénères de la condition MS $(+7,62)$ et celles de la condition GC $(+5,86)$.

Entre le pré-test et le post-test différé, RMANOVA présente les mêmes effets que ceux mentionnés précédemment.

\section{Discussion}

La présente étude se proposait de modéliser un programme de Formation au Managérat Réciproque entre Pairs (FMRP) dans l'attente de bénéfices moteurs supérieurs chez des élèves débutants impliqués dans un cycle de tennis de table. Par ailleurs, des différences liées au genre étaient envisagées. Conformément à la littérature et à nos hypothèses, le programme FMRP a provoqué des performances motrices plus élevées comparativement à celles obtenues dans le cadre d'un managérat spontané (MS) ou d'une pratique seule (GC). Ainsi, relativement à l'atteinte des zones latérales et de l'exécution correcte de smash lors des renvois (score d'efficacité de l'action) ou des prises de décision quant au type de frappe à produire (score de pertinence du choix), les participants de la condition MF se sont montrés plus performants comparativement à leurs homologues des deux autres conditions d'entraînement. En revanche, contrairement aux travaux de Darnis et al. (2005 a), la compétence motrice affichée par les élèves ayant interagi spontanément et celle manifestée par les élèves ayant pratiqué seuls sont identiques. Sur ce point, la nature différente de la tâche utilisée pour l'expérimentation s'avère éclairante. Inspirée d'une situation de sport collectif obligeant les élèves à coopérer pour réussir (2x1 en handball), la tâche employée par Darnis et al. (2005 a) est plus propice à l'émergence de bénéfices chez des participants ayant l'occasion d'interagir, indépendamment de la présence d'une formation. L'importance des échanges verbaux dans les sports collectifs comparativement aux sports individuels à d'ailleurs fait l'objet d'études récentes (Lafont et al., 1999 ; Lafont, Proeres et Vallet, 2007). Dans le cas présent, cette divergence ne fait que renforcer l'idée selon laquelle le recours à une formation des apprenants à interagir avec un pair est une condition d'efficacité coopérative essentielle. Plus que facilitant, le programme FMRP s'avère ici indispensable à l'émergence de bénéfices moteurs dans le cadre d'un managérat réciproque entre pairs. Ce postulat est confirmé par l'analyse des résultats relatifs aux progrès (RMANOVA).

S'agissant d'un éventuel effet du genre, les résultats sont en partie conformes avec les études antérieures. Ainsi, conformément aux études de Darnis et al. (2005a et b), les scores relatifs au niveau de compétence motrice obtenus par les garçons sont le plus souvent supérieurs à ceux des filles. Sur ce point, deux explications peuvent être 
envisagées. D'une part, il est possible d'avancer un argument en matière de socialisation différentielle, ainsi, on peut penser que les garçons ont été plus souvent que les filles, dès leur plus jeune âge, confrontés à des tâches d'anticipation coïncidence (Anderson, Gebhart, Pease and Rupnow, 1983). D'autre part, on peut envisager le fait que, malgré les précautions prises lors de sa conception et la faible connotation de genre du tennis de table, la tâche expérimentale ait été perçue comme une situation comparative ou compétitive par les participants. Ainsi, l'idée selon laquelle le but de la tâche était de mieux performer que son camarade ou de prouver sa compétence à l'expert aurait favorisé l'émergence d'une compétence motrice plus élevée chez les garçons. En cela, ces résultats invitent les futures recherches à la plus grande vigilance quant au choix de l'activité et de la tâche motrice pour évaluer le plus objectivement possible un éventuel effet du genre.

Par ailleurs, l'absence de différence significative quant aux progrès effectués en situation concrète de match $(\mathrm{MD})$ doit être interprétée avec prudence, le calcul de cette variable ne permettant pas une évaluation juste des progrès réalisés pour l'ensemble des participants. En effet, ayant généralement accédé aux tables les plus fortes à l'issue de MD1, les garçons se sont trouvés dans l'impossibilité de progresser aussi significativement que les filles. Cette remarque devrait amener les études futures à réfléchir à l'opérationnalisation d'une nouvelle variable.

Relativement aux différences liées à l'interaction de la condition d'entraînement et du genre, l'analyse des résultats montre que les filles ayant bénéficié de l'aide éclairée d'un pair réalisent des performances et des progrès moteurs plus élevés que ceux obtenus par leurs congénères ayant interagi spontanément ou ayant pratiqué seules. Ces résultats sont consistants avec ceux mentionnés dans une étude récente conduite dans le cadre d'acquisitions de tâches motrices à connotation féminine (tâches gymniques, Legrain et Heuzé, 2005). Ainsi, les auteurs soulignent une sensibilité plus importante des filles à la structuration de leur environnement social (RPT). En cela, notre étude étend la validité de leurs propos à l'acquisition d'habiletés motrices moins connotées sexuellement et présente explicitement la nature de la structuration utilisée (FMRP).

\section{Conclusion}

Le recours à une procédure d'interaction réciproque de tutelle ne semble pas, à elle seule, influencer un apprentissage au choix décisionnel en tennis de table. En revanche, accompagnée de la mise en place d'un programme de formation des apprenants, cette stratégie d'apprentissage coopératif devient alors porteuse de progrès. Ainsi, on constate 
un gain d'efficacité dans la réalisation de la tâche-cible uniquement chez les participants ayant bénéficié de l'aide éclairée d'un pair. Déjà perçue par de précédentes études comme une condition d'efficacité coopérative essentielle, la préparation des élèves à interagir avec un pair devient ici indispensable dans l'attente d'une véritable « coconstruction des savoirs ».

Par ailleurs, en continuité avec l'étude récente de Legrain et Heuzé (2005), la présente étude confirme l'existence d'une sensibilité plus importante des filles à la structuration de leur environnement social. Ainsi, de manière originale, elle étend la validité des conclusions de ces auteurs à l'acquisition d'habiletés motrices moins connotées sexuellement tout en décrivant explicitement la nature de la structuration utilisée (FMRP).

En outre, les conclusions encourageantes de ce travail invitent à poursuivre la réflexion. Deux axes de recherche semblent prioritaires. D'une part, eu égard aux résultats de travaux antérieurs mentionnant l'obtention souvent conjointe de bénéfices cognitifs, motivationnels, sociaux et moteurs consécutivement à la mise en place de stratégies d'AC (Ward \& Lee, 2005), la mesure des effets du programme FMRP sur un large panel de variables dépendantes parait pertinente. D'autre part, en accord avec la littérature (Darnis et al., 2005 b ; Lafont et al., 2005 ; Legrain et al., 2003), il serait intéressant d'envisager une analyse interlocutoire des interactions dyadiques permettant notamment d'évaluer l'impact de la FMRP sur les compétences managériales des élèves.

\section{Bibliographie}

Anderson, D.F., Gebhart, J.A., Pease D.G., \& Rupnow,A.A. (1983). Effects of age, sex, and placement of a model on children's performance on a ball-striking task. Perceptual and Motor Skills, 57, 1187-1190.

Arripe-Longueville (d'), F., Gernigon, C., Huet, M., Cadopi, M., \& Winnykamen, F. (2002). Peer tutoring in a physical education setting: Influence of tutor skill level on novice learners' motivation and performance. Journal of Teaching in Physical Education, 22, 105-123.

Bandura, A. (1997). Self-efficacy: The exercice of control. New York: Freeman.

Brunelle, J., Drouin, D., Godbout, P., \& Tousignant, M. (1988). La supervision de l'intervention en activité physique. Montréal: Gaëtan Morin Éditeur.

Charlesworth, W.R., \& Dzur, D. (1987). Gender comparisons of preschooler's behaviour and resource utilization in group problem solving. Child Development, 58, 191200. 
Darnis, F., Lafont, L., \& Menaut, A. (2005a). A social-constructivist approach in physical education : Influence of dyadic interactions on tactical choices in an instructional team sport setting. European Journal of Psychology of Eduction, 20, (2), 171-184.

Darnis, F., Lafont, L., \& Menaut, A. (2005b). Interactions dyadiques et niveau opératoire pour la construction de stratégies en handball chez des participants de 11-12 ans. Revue européenne de psychologie appliquée, 55, 255-265.

Delisle, H. (1999). Tennis de table - Technique, tactique et didactique. Paris : Revue EP.S

Duran, D., \& Monereo, C. (2005). Styles and sequences of cooperative interaction in fixed and reciprocal peer tutoring. Learning and Instruction, 15, 179-199.

Ellis, S., \& Rogoff, B. (1982). The strategies and efficacy of child versus adult teachers. Child development, 53, 730-735.

Fantuzzo, J.W, Riggio, R.E. Connely, S., \& Dimeff, L.A. (1989). Effects of reciprocal peer tutoring on academic achievement and psychological adjustment: A component analysis. Journal of Educational Psychology, 81(2), 173-177.

Fantuzzo, J.W., \& Ginsburg-Block, M. (1997). Reciprocal Peer tutoring: an analysis of teacher and student interaction as a function of training and experience. School Psychology Quarterly, 12(2), 134-149.

Fantuzzo, J.W., King, A.K., \& Heller, L.R. (1992). Effects of reciprocal peer tutoring on mathematics and school adjustment: A component analysis. Journal of Educational Psychology, 3, 331-339.

Johnson, D.W., \& Johnson, R.T. (1989). Cooperation and competition: Theory in the research. Edina, MN: Interaction Book CO.

Johnson, D.W., Johnson, R.T., \& Smith, A. (1998). Cooperative learning returns to College: What evidence is there that it works? Change, 27-35.

Jonhson, M., \& Ward, P. (2001). Effects of classwide peer tutoring on correct performance of striking skills in $3^{\text {rd }}$ grade physical education. Journal of Teaching in Physical Education, 20, 247-263.

Kagan, S. (1990). The structural approach to cooperative learning. Educational Leadership, 47, 2-16.

Lafont, L., Cicero, C., Vedel, A. \& Viala, M. (2005). Apports de la psychologie sociale à l'intervention en EPS : rôle des interactions tutorielles et des "coping" modèles. @ejrieps, 8, Juillet 2005.

Lafont, L, Proeres, M., Burvingt, F., Poitreau, D., \& Menaut, A. (1999). Les situations de coopération en EPS. Communication présentée aux Actes du Congrès International AFRAPS EDMP. Louvain-La Neuve. 
Lafont, L., Proeres, M. \& Vallet, C. (2007). Cooperative group learning in a team game: role of verbal exchanges between peers. Social Psychology of Education, 10, 1, 93-113.

Lafont, L., \& Winnykamen, F. (1999). Cooperation and competition in children and adolescents. In Y. Vauden Auweele, F. Bakker, S. Biddle, M. Durand, \& R. Seiler (Eds.), Psychology for educators (pp. 379-404). Champaign, IL: Human Kinetics.

Legrain, P., Arripe-Longueville (d'), F. \& Gernignon, C. (2003). The influence of trained peer tutoring on tutors' motivation and performance in a French boxing setting. Journal of Sports Sciences, 21, 7, 159-160.

Legrain, P., \& Heuzé, J.P. (2005). Intégration d'une tutelle réciproque entre pairs dans un dispositif collectif d'apprentissage en gymnastique: étude de variables médiatrices. Symposium présenté aux Actes du Congrès International de la SFPS. Reims.

Lorence, B. (2002). Le tutorat entre enfants. Association des enseignants et chercheurs en Sciences de l'Education. http://aecse2001.univ-lille.fr/cdrom/Coms/Lorence.htm

Sève, C. (2003). Le tennis de table en situation. Paris : Revue EP.S.

Slavin, R.E. (1995). Cooperative learning: Theory, research and practice. (2nd ed.) Boston: Allyn \& Bacon.

Ward, P., \& Lee, M-A. (2005). Peer-assisted learning in physical education : A review of theory and research. Journal of Teaching in Physical Education, 24, 205-225.

Wiegmann, D.A., Dansereau, D.F. \&, Patterson, M.. (1992). Cooperative learning: Effects of role-playing and ability on performance. Journal of Experimental Education, 60, 109-116.

Winnykamen, F. (1996). Expert et/ou tuteur : les comparaisons des dyades adulte/enfant et enfant/enfant peuvent-elles éclairer le processus de guidage? Revue de Psychologie de l'Education, 2, 13-35. 